\title{
Habilidad gráfica y numérica en estudiantes de medicina de pre y posgrado de una universidad privada
}

\author{
Guiliana Mas', Tania Tello1, Pedro Ortiz¹, Dafina Petrova² y Rocío García-Retamero² \\ ${ }^{1}$ Instituto de Gerontología, Universidad Peruana Cayetano Heredia, Lima, Perú; ${ }^{2}$ Universidad de Granada, Granada, España.
}

\section{Resumen}

Introducción: Los médicos y estudiantes de medicina necesitan comprender los datos numéricos y gráficos sobre salud para proveer información correcta a los pacientes. Objetivo: Determinar las habilidades gráficas y numéricas en estudiantes y residentes de medicina de una universidad privada de Lima, Perú. Método: Estudio descriptivo, transversal. Se aplicaron las escalas de Habilidad Numérica Objetiva, de Habilidad Numérica Subjetiva y de Habilidad Gráfica a estudiantes de medicina de los dos últimos años de la carrera de medicina y a residentes de medicina. Resultados: De 169 participantes, $52.07 \%$ fueron estudiantes de sexto año, $18.34 \%$ de séptimo y $29.58 \%$ residentes. La media en la habilidad numérica objetiva fue de 7.34, en la subjetiva de 34.12 y en la habilidad gráfica de 10.35. De la regresión lineal múltiple se obtuvo que las medias más altas en las escalas de Habilidad Numérica Subjetiva y Objetiva estuvieron asociadas con el sexo masculino y la capacitación sobre metodología en investigación $(p<0.05)$. En la Escala de Habilidad Gráfica, las medias más altas estuvieron asociadas con el sexo masculino y menor edad $(p<0.05)$. Conclusión: Las medias de las escalas de Habilidad Gráfica y Habilidad Numérica en estudiantes de medicina fueron altas.

PALABRAS CLAVE: Habilidad numérica. Habilidad gráfica. Estudiantes de medicina.

\begin{abstract}
Introduction: Physicians and medical students need to understand numerical and graphical health data in order to provide patients with correct information. Objective: The graphical and numerical skills of medical students and residents from a private university in Lima, Peru, were determined in this investigation. Method: Cross-sectional, descriptive study. The Objective Numeracy, Subjective Numeracy and Graph Literacy Scales were applied to medical students at their two final years of medical school and to medical residents. Results: Of 169 participants, 52.07\% were sixth-year and $18.34 \%$ were seventh-year students and $29.58 \%$ were residents. Mean objective numeracy score was 7.34 , mean subjective numeracy was 34.12 and mean graph literacy was 10.35. A multiple linear regression analysis showed that Subjective and Objective Numeracy Scales highest means were associated with the male gender and training on research methodology $(p<0.05)$. Graph Literacy Scale highest means were associated with the male gender and younger age ( $p$-value $<0.05)$. Conclusion: Numeracy and Graph Literacy Scales mean scores were high in medical students.
\end{abstract}

KEY WORDS: Numeracy. Graph literacy. Medical students.

Correspondencia:

Guiliana Mas

E-mail: guiliana.mas.u@upch.pe
Fecha de recepción: 01-06-2017

Fecha de aceptación: 25-06-2017

DOI://dx.doi.org/10.24875/GMM.17003024
Gac Med Mex. 2018;154:209-216

Disponible en PubMed

www.gacetamedicademexico.com 


\section{Introducción}

La habilidad para comprender gráficos, así como el entendimiento de expresiones numéricas de riesgo como probabilidades, porcentajes y frecuencias es esencial en el área médica. Los médicos y estudiantes de medicina necesitan comprender y entender la importancia de la información numérica y gráfica relacionada con temas de salud (por ejemplo, riesgos de diferentes enfermedades y tratamientos), para transmitir información adecuada a los pacientes. ${ }^{1-3}$

Para tomar una decisión efectiva, el personal de salud debe entender los riesgos y beneficios de diferentes tratamientos médicos, pruebas diagnósticas y modificaciones en los estilos de vida; sin embargo, diversas investigaciones indican que no siempre comprende conceptos numéricos y gráficos, habilidades esenciales para el entendimiento y comunicación en los temas de salud. ${ }^{4-7}$

Para la investigación de estas habilidades se han desarrollado escalas en diversos países como Estados Unidos, Alemania y España, ${ }^{8-10}$ las cuales cumplen con propiedades psicométricas apropiadas para ser aplicadas en diversos escenarios clínicos y de investigación. ${ }^{11,12}$

La Escala Numérica Objetiva llevada a cabo por Lipkus et al. ${ }^{13}$ está enfocada en llevar a cabo operaciones matemáticas simples con porcentajes y proporciones, convertir porcentajes a proporciones, proporciones a porcentajes y probabilidades a proporciones, todas de uso común en la comunicación para toma de decisiones y la explicación de riesgos-beneficios de diversos procesos. Esta escala consta de nueve reactivos que han sido aplicados en forma satisfactoria en personas con baja y alta habilidad numérica en Estados Unidos, Alemania y España. Además, existe una Escala Numérica Subjetiva, que mide las habilidades numéricas mediante una autoevaluación de los participantes, en la que se indica, por ejemplo, en qué grado son buenos para hacer cálculos con porcentajes. ${ }^{14,15}$ Esta escala ha demostrado correlación alta con la Escala Numérica Objetiva.

Galesic y García Retamero ${ }^{16}$ desarrollaron una nueva escala de literatura gráfica con el objetivo de investigar las habilidades de leer y comprender gráficos en el contexto de decisiones médicas y ser lo suficientemente breve para usar a diario en la práctica clínica. Esta escala fue validada y conducida en muestras nacionales probabilísticas en Alemania y Estados Unidos y ha sido utilizada satisfactoriamente en España. ${ }^{14}$
El presente estudio tiene como objetivo determinar las habilidades gráficas y numéricas de los estudiantes y residentes de medicina de pre y posgrado de una universidad privada, mediante la validación y posterior aplicación de las tres escalas: de habilidad numérica objetiva, de habilidad numérica subjetiva y de habilidad gráfica, puesto que es una área no investigada en Perú, con lo que se demostró el impacto que conlleva una deficiente comunicación e información de los pacientes en la toma compartida de decisiones en medicina.

\section{Método}

\section{Lugar, diseño y participantes del estudio}

Se llevó a cabo un estudio descriptivo, transversal y exploratorio de una población de estudiantes de medicina de sexto y séptimo año de la Facultad de Medicina Alberto Hurtado, de la Universidad Peruana Cayetano Heredia, que se encontraban en rotación por los servicios de medicina interna de los hospitales Arzobispo Loayza y Cayetano Heredia, hospitales públicos de tercer nivel del Ministerio de Salud del Perú, ubicados en el área norte de la ciudad de Lima; así como de residentes de medicina del primer al cuartoaño de todas las especialidades de los hospitales mencionados, previo consentimiento informado. El estudio se llevó a cabo en 2014.

Se les aplicaron las escalas de habilidad numérica objetiva, de habilidad numérica subjetiva y de habilidad gráfica, durante un tiempo promedio de veinte minutos. Además, se llenó una ficha de recolección de datos sociodemográficos de cada participante.

El muestreo fue por conveniencia, de acuerdo con la presencia de los alumnos en los servicios de medicina interna en las sedes hospitalarias en el periodo de la evaluación. La muestra de alumnos de sexto año (externos) fue de 88 de un total de 100; 12 (12\%) se encontraban en rotaciones fuera del país. La muestra de alumnos de séptimo año (internos) fue de 31 , de un total de 114; 83 (73\%) estuvieron fuera de la rotación de medicina interna. La muestra de residentes de medicina fue de 50 de un total de 90; 40 (45\%) se encontraban de vacaciones o en rotaciones fuera de la sede o del país, como se muestra en la Figura 1.

\section{Variables}

Se recolectaron datos de los participantes que incluyeron edad, sexo, lugar de procedencia, año 


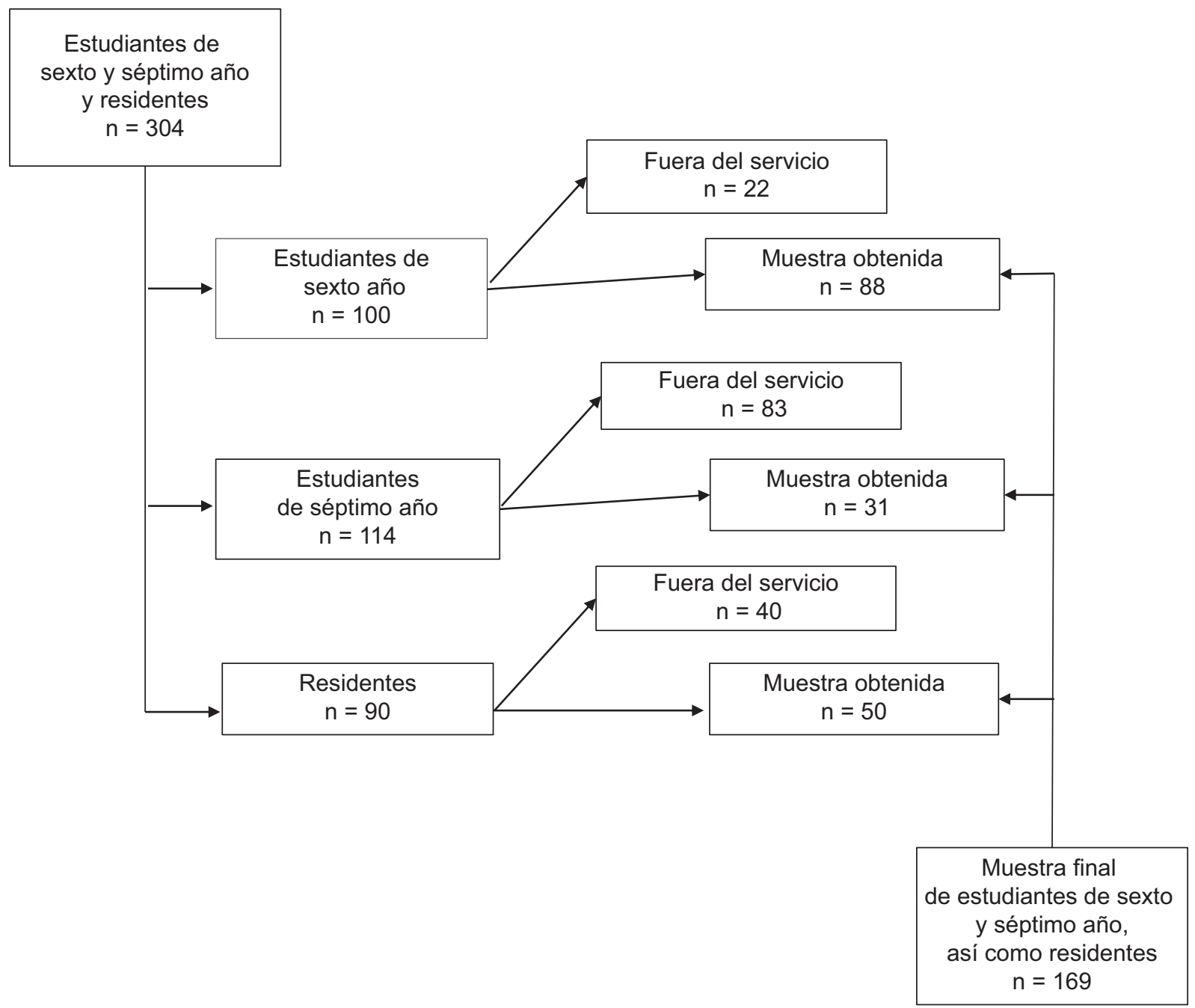

Figura 1. Selección de participantes en los servicios de medicina interna de las sedes hospitalarias.

académico, año egresado de la facultad de medicina, conocimiento previo en metodología o estadística y conocimiento del idioma inglés.

La Escala de Habilidad Numérica Objetiva consiste en nueve reactivos, en los que se lleva a cabo operaciones matemáticas simples usando porcentajes y proporciones. La Escala de Habilidad Numérica Subjetiva contiene siete reactivos cuyas respuestas no son correctas o incorrectas; se obtiene la valoración media de todos reactivos. La Escala de Habilidad Gráfica está integrada por 13 reactivos y mide tres habilidades relacionadas con la comprensión gráfica: ${ }^{16}$

- Capacidad para leer los datos, encontrar la información específica en el gráfico.

- Capacidad para leer entre los datos, encontrar la relación en los datos como se muestran en los gráficos.
- Capacidad para leer más allá de los gráficos o hacer inferencias y predicciones en los datos.

\section{Ética}

Este estudio fue aprobado por el Comité de Ética de la Universidad Peruana Cayetano Heredia.

\section{Análisis estadístico}

Las variables cuantitativas son descritas con media y desviación estándar y las variables cualitativas con frecuencia y porcentajes. Para la evaluación de la Escala Numérica Objetiva, las respuestas fueron analizadas mediante puntuaciones (sumando el número de respuestas correctas, con rango posible de valores de 0 a 9). La Escala Numérica Subjetiva contiene siete reactivos, cuyas respuestas no son correctas o incorrectas, se obtiene la valoración media de todos 
reactivos (rango de valores de 6 a 42). La Escala Gráfica Objetiva comprende 13 reactivos; se procedió a sumar el número de respuestas correctas (rango de valores de 0 a 13).

Se llevaron a cabo análisis de confiabilidad y validez de construcción en el contexto peruano para cada escala, siguiendo el procedimiento adoptado por los autores de las versiones originales. ${ }^{13-16}$ Para todas las escalas se computó $\alpha$ de Cronbach, la correlación media entre reactivos y la correlación media entre cada reactivo y la puntuación total. En la Escala Numérica Subjetiva se esperaban correlaciones más altas conforme el tipo de escala (autoinforme). En la Escala Numérica Objetiva se esperaba correlaciones más bajas que reflejaran la contribución original de cada reactivo a la capacidad discriminatoria de las escalas. ${ }^{16}$ Se llevó a cabo análisis factorial confirmatorio de la Escala Numérica Objetiva para validar la estructura unifactorial establecida de la escala. ${ }^{13}$ Las Escala Numérica Subjetiva y la Escala Gráfica Objetiva se evaluaron mediante análisis factorial exploratorio, dado que no se evaluó su estructura en sus versiones originales..$^{15,16} \mathrm{La}$ validez de cada escala se valoró adicionalmente mediante correlaciones con las otras escalas y los factores sociodemográficos relacionados en la literatura.

Al final se llevó a cabo un análisis de regresión lineal múltiple para establecer factores predictores respecto a cada escala. Las variables dependientes serán respectivamente Escala Numérica Objetiva, Escala Numérica Subjetiva, Escala de Habilidad Gráfica. Las variables independientes que se ingresaron en el estudio fueron la edad, el sexo, la capacitación previa de metodología y el grupo de alumnos (pregrado y posgrado de medicina).

\section{Resultados}

De los 169 participantes, $52.07 \%$ (88/169) fue externo (estudiantes de sexto año de medicina), $18.34 \%$ (31/169) internos (estudiantes de séptimo año de medicina) y $29.58 \%(50 / 169)$ residentes de medicina de la Universidad Peruana Cayetano Heredia, que rotaban en los hospitales nacionales Arzobispo Loayza y Cayetano Heredia. Las características de los alumnos de sexto y séptimo año de medicina y de los residentes de medicina se muestran en la Tabla 1.

De los residentes participantes, $22 \%$ (11) fue de la especialidad de medicina interna, $10 \%$ (5) de nefrología y $10 \%$ (10) de medicina intensiva. Respecto a los años académicos, $60 \%$ (30) cursaba el primer año, $26 \%$ (13) el segundo año y $14 \%$ (siete) el tercer año de cada especialidad; 68 \% (34) de los residentes egresó de la Facultad de Medicina entre 2006 y 2012.

En la Escala Objetiva Numérica se obtuvo una media \pm desviación estándar de $7.34 \pm 1.51$, en la Escala Subjetiva Numérica de $34.12 \pm 4.87$ y en la Escala de Habilidades Gráficas de $10.35 \pm 1.93$.

\section{Escala Objetiva Numérica}

El reactivo 6 fue excluido del análisis de confiabilidad porque todos los participantes formularon una respuesta correcta. La escala mostró un $\alpha$ de Cronbach cuestionable de 0.61 . La correlación entre reactivos tuvo una media de $r=0.161$ y $r=0.317$ entre cada reactivo y la puntuación total, lo que mostró que cada reactivo mide un aspecto distinto de las habilidades numéricas. El modelo unifactorial mostró buen ajuste con $\chi^{2}=24.42, p=0.224$ y RMSEA $=0.037(0.05$ como corte aceptable). ${ }^{17}$

\section{Escala Subjetiva Numérica}

La escala mostró $\alpha$ de Cronbach buena de 0.81. La correlación media entre reactivos fue $r=0.392$ y la correlación media entre cada reactivo y la puntuación total fue $r=0.555$, lo que mostró alta consistencia interna del autoinforme. El análisis factorial exploratorio identificó dos factores (eigenvalue > 1), lo que explicó $64 \%$ de la varianza. El primer factor estuvo compuesto por los reactivos 1,2 y 3, lo que reflejó la autoevaluación de las habilidades numéricas y el segundo factor estuvo compuesto por los reactivos 4, 5, 6 y 7, lo que indicó la preferencia por la información numérica. Estos factores son consistentes con el concepto teórico de la escala. ${ }^{18}$ Se guardaron las puntuaciones de cada factor con rotación varimax como variables adicionales. No se observaron diferencias entre los resultados obtenidos con las variables adicionales y la puntuación original de la escala, por lo cual se demuestran solo los resultados de la puntuación original.

\section{Escala Objetiva Gráfica}

La escala mostró $\alpha$ de Cronbach de 0.66 , aproximada a lo aceptable. La correlación media entre los reactivos fue $r=0.136$ y la correlación media entre cada reactivo y la puntuación total fue $r=0.297$, lo que traduce que cada reactivo mide un aspecto distinto de las habilidades gráficas. Las medias y 
Tabla 1. Características demográficas de los alumnos sexto, séptimo y residentes

\begin{tabular}{|c|c|c|c|c|c|c|}
\hline \multirow[t]{2}{*}{ Variable } & \multicolumn{6}{|c|}{ Alumnos } \\
\hline & \multicolumn{2}{|c|}{$\begin{array}{l}\text { Sexto } \\
(n=88)\end{array}$} & \multicolumn{2}{|c|}{$\begin{array}{l}\text { Séptimo } \\
(n=31)\end{array}$} & \multicolumn{2}{|c|}{$\begin{array}{l}\text { Residentes } \\
\quad(n=50)\end{array}$} \\
\hline \multirow[t]{2}{*}{ Edad (media $\pm \mathrm{DE})$} & \multicolumn{2}{|c|}{$23.13 \pm 1.39$} & \multicolumn{2}{|c|}{$23.80 \pm 1.27$} & \multicolumn{2}{|c|}{$31.92 \pm 6.40$} \\
\hline & $\mathrm{n}$ & $\%$ & $\mathrm{n}$ & $\%$ & $\mathrm{n}$ & $\%$ \\
\hline \multicolumn{7}{|l|}{ Sexo } \\
\hline Hombres & 44 & 50 & 20 & 64.32 & 26 & 52 \\
\hline Mujeres & 44 & 50 & 11 & 35.48 & 24 & 48 \\
\hline \multicolumn{7}{|l|}{ Lugar de procedencia } \\
\hline Lima & 62 & 70.45 & 24 & 77.42 & 26 & 52 \\
\hline Provincia & 7 & 7.98 & 7 & 19.37 & 24 & 48 \\
\hline \multicolumn{7}{|l|}{ Hospital de procedencia } \\
\hline HNAL & 0 & 0 & 20 & 64.52 & 21 & 42 \\
\hline $\mathrm{HNCH}$ & 88 & 100 & 11 & 35.48 & 29 & 58 \\
\hline \multicolumn{7}{|l|}{ Conocimiento inglés } \\
\hline No sabe & 2 & 2.27 & 0 & 0 & 0 & 0 \\
\hline Lee & 8 & 9.09 & 2 & 6.45 & 14 & 28 \\
\hline Lee y escribe & 6 & 6.82 & 5 & 16.13 & 18 & 36 \\
\hline Lee, escribe y habla & 69 & 78.41 & 24 & 77.42 & 17 & 34 \\
\hline \multicolumn{7}{|l|}{$\begin{array}{l}\text { Capacitación previa en } \\
\text { metodología }\end{array}$} \\
\hline Sí & 67 & 76.14 & 16 & 51.61 & 15 & 30 \\
\hline No & 18 & 20.45 & 14 & 45.16 & 34 & 68 \\
\hline
\end{tabular}

HNAL, Hospital Nacional Arzobispo Loayza; HNCH, Hospital Nacional Cayetano Heredia

desviaciones estándar de los reactivos fueron las siguientes: capacidad para leer datos $3.85 \pm 42$ (min. 2, máx. 4), capacidad para leer entre los datos $3.33 \pm 0.89$ ( $\min .0$, máx. 4), capacidad de leer más allá de los datos $3.18 \pm 1.10$ (min. 0 , máx. 5).

El análisis factorial exploratorio identificó cinco factores (eigenvalue > 1) que explicaron $60 \%$ de la varianza. El mayor porcentaje de varianza fue explicado por el primer factor (habilidad gráfica, $22 \%$ ) con cargas factoriales medias-altas (de 0.271 a 0.619 ) de todos los reactivos. Se buscó una interpretación del resto de los factores aplicando varias rotaciones. Cada factor estaba compuesto por un par de reactivos relacionados con el tipo de gráfica o tipo de respuesta de los reactivos. Concluimos que estos factores reflejan principalmente la variación de medición común en lugar de construcciones teóricas significativas, un resultado típico en este tipo de escalas. ${ }^{13}$

Se observaron correlaciones significativas de tamaño medio entre las tres habilidades (numérica objetiva-numérica subjetiva $r=0.44, p<0.001$; numérica objetiva-gráfica objetiva $r=0.36, p<0.001$; y numérica subjetiva-gráfica objetiva $r=0.24, p=0.002$ ), lo que demostró que las tres escalas miden habilidades distintas, pero relacionadas entre sí.

\section{Factores relacionados con las habilidades numéricas y gráficas}

En la Tabla 2 se muestran los resultados por grupo de alumnos, sexo y capacitación previa en metodología; los mayores resultados los presentaron los alumnos de sexto año (externos), los varones y los alumnos que habían recibido capacitación en metodología durante su enseñanza.

Se llevó a cabo un análisis de regresión lineal múltiple, en el que las variables dependientes fueron la Escala Numérica Objetiva, la Escala Numérica Subjetiva y la Escala de Habilidades Gráficas. Las variables independientes ingresadas al modelo fueron la edad, el sexo, el conocimiento en metodología y el grupo de alumnos (variable dummy). En las habilidades numérica subjetiva y numérica objetiva, los estudiantes varones y quienes habían recibido capacitación en metodología obtuvieron los resultados más altos $(p<0.05)$. En cuanto a la habilidad gráfica, las medias más altas se asociaron con el sexo masculino y menor edad ( $p<0.05)$, sin embargo, no hubo relación con la capacitación previa en metodología. Los internos tuvieron una habilidad gráfica mayor que 
Tabla 2. Resultados promedios de las escalas por grupo de alumnos, sexo y conocimiento en metodología

\begin{tabular}{lccc}
\hline Variable & $\begin{array}{c}\text { Escala } \\
\text { Numérica Objetiva }\end{array}$ & $\begin{array}{c}\text { Escala } \\
\text { Numérica Subjetiva }\end{array}$ & $\begin{array}{c}\text { Escala } \\
\text { Habilidad Gráfica }\end{array}$ \\
\hline Grupo de alumnos & & & \\
$\quad$ Alumnos de sexto año & $7.72 \pm 1.25$ & $35.14 \pm 4.98$ & $10.54 \pm 1.74$ \\
$\quad$ Alumnos de séptimo año & $7.16 \pm 1.55$ & $33.67 \pm 3.96$ & $11.22 \pm 1.30$ \\
$\quad$ Residentes & $6.71 \pm 1.74$ & $32.44 \pm 4.82$ & $9.40 \pm 2.26$ \\
$\quad$ Total & $7.34 \pm 1.51$ & $34.12 \pm 4.87$ & $10.35 \pm 1.93$ \\
Sexo & & & $10.67 \pm 1.68$ \\
$\quad$ Hombres & $7.57 \pm 1.46$ & $35.43 \pm 4.63$ & $10.00 \pm 2.13$ \\
$\quad$ Mujeres & $7.06 \pm 1.53$ & $32.61 \pm 4.73$ & $10.35 \pm 1.93$ \\
$\quad$ Total & $7.34 \pm 1.51$ & $34.12 \pm 4.87$ & $10.01 \pm 2.11$ \\
Capacitación previa en metodología & & & $10.51 \pm 1.81$ \\
$\quad$ No & $6.45 \pm 1.76$ & $32.11 \pm 4.71$ & $10.32 \pm 1.94$ \\
$\quad$ Sí & $7.87 \pm 1.05$ & $35.44 \pm 4.61$ & $34.16 \pm 4.91$ \\
$\quad$ Total & $7.33 \pm 1.53$ & & \\
\hline
\end{tabular}

los residentes (marginalmente significativa). Los resultados se muestran en la Tabla 3.

\section{Discusión}

En el presente estudio se observó que los 169 alumnos participantes del sexto, séptimo y residentes de la Facultad de Medicina de la Universidad Peruana Cayetano Heredia mostraron promedios de resultados de escalas numéricas y gráficas altas: $82 \%$ (7.34/9) con habilidad numérica y $80 \%(10.35 / 13)$ con habilidad gráfica ( $20 \%$ de error). Algunos estudios reportan $55 \%$ de error en las pruebas de habilidad numérica en estudiantes de enfermería ${ }^{19}$ y que $61 \%$ de los estudiantes de medicina interpretaron adecuadamente datos cuantitativos aun cuando respondieron correctamente un cuestionario de habilidad numérica. ${ }^{20}$ En estudiantes de medicina se registró que solo $69 \%$ respondió correctamente las pruebas de habilidad numérica ${ }^{21}$ y la comprensión correcta de estadísticos en literatura médica fue solo de $41.4 \%$ en residentes de medicina. ${ }^{22}$

En general, los análisis de confiabilidad y validez de construcción indicaron que las escalas son medidas válidas de las habilidades numéricas y gráficas. Sin embargo, se identificaron algunas diferencias importantes. Primero, ambas escalas objetivas mostraron índices de confiabilidad menos satisfactorios de los que se habían encontrado en su validación inicial. Estos resultados se pueden deber a sensibilidad cultural de las escalas en la muestra peruana 0 , lo que consideramos más probable, a las diferencias en habilidad entre la población general y los profesionales de medicina. ${ }^{23}$ Ambas escalas se han validado en población general, incluyendo personas con bajos niveles de educación, lo cual dificulta la comparación con la muestra de nuestro estudio, compuesta por participantes con nivel de educación alto que consiguen puntuaciones altas en ambas escalas. Por ejemplo, un reactivo de la Escala Numérica Objetiva no mostró discriminación y fue respondido correctamente por todos los participantes. Encontramos resultados parecidos con la Escala Gráfica Objetiva: Ios reactivos que miden el nivel de comprensión gráfica más básico - capacidad para leer los datos (encontrar la información específica en el gráfico) - fueron respondidos correctamente por la mayoría de los participantes y ofrecieron poca capacidad discriminatoria. Esto significa que las escalas objetivas administradas en este estudio tienen menos capacidad discriminatoria y una confiabilidad mejorable en poblaciones con altas habilidades, como los estudiantes de medicina. Investigaciones futuras deberán adaptar mejor los instrumentos al contexto peruano y al contexto médico profesional. Existe otra escala numérica objetiva, Berlin Numeracy Test, validada para su uso en poblaciones con altos niveles de educación y en profesionales de medicina. Ese instrumento tiene la ventaja de ser más corto (dos a tres preguntas) y está disponible en español (http://www.riskliteracy.org/). En cuanto a la Escala Objetiva de Habilidades Gráficas, en estudiantes o profesionales de medicina recomendamos el uso de una versión abreviada que incluye solo los reactivos que miden las dos competencias gráficas de nivel más alto - capacidad para leer entre los datos y capacidad para leer más allá de los datos-. Esta versión abreviada podría acortar significativamente el tiempo necesario para la evaluación eliminando reactivos no discriminatorios en estas poblaciones. 
Tabla 3. Análisis de regresión lineal múltiple de las habilidades numéricas y gráficas en alumnos de sexto, séptimo y residentes de la Universidad Peruana Cayetano Heredia

\begin{tabular}{|c|c|c|c|c|c|c|c|c|c|c|}
\hline \multirow[t]{2}{*}{ Variables } & \multicolumn{2}{|c|}{ Sexo } & \multicolumn{2}{|c|}{ Edad } & \multicolumn{2}{|c|}{ Capacitación previa en metodología } & \multicolumn{2}{|c|}{ Externos } & \multicolumn{2}{|c|}{ Internos } \\
\hline & Coef. $\beta$ & $\mathrm{p}$ & Coef. $\beta$ & $\mathrm{p}$ & Coef. $\beta$ & $\mathrm{p}$ & Coef. $\beta$ & p & Coef. $\beta$ & $\mathrm{p}$ \\
\hline $\begin{array}{l}\text { Escala Numérica Objetiva } \\
r^{2}=0.22\end{array}$ & -0.16 & 0.02 & -0.01 & 0.31 & 0.38 & 0.00 & 0.09 & 0.46 & -0.02 & 0.84 \\
\hline $\begin{array}{l}\text { Escala Numérica Subjetiva } \\
\mathrm{r}^{2}=0.18\end{array}$ & -0.28 & 0.00 & -0.02 & 0.82 & 0.24 & 0.00 & 0.17 & 0.16 & 0.04 & 0.67 \\
\hline $\begin{array}{l}\text { Escala de Habilidad Gráfica } \\
r^{2}=0.14\end{array}$ & -0.18 & 0.01 & -0.25 & 0.02 & 0.03 & 0.62 & 0.07 & 0.58 & 0.19 & 0.07 \\
\hline
\end{tabular}

Si bien los resultados incluyen dos poblaciones en etapas distintas de entrenamiento, alumnos de pregrado de la carrera de medicina y médicos residentes en especialización, es importante resaltar las diferencias dado que los programas de especialización no deberían descuidar el entrenamiento y capacitación de competencias en comunicación de decisiones y riesgos mediante estrategias que ya han sido descritas,$^{15}$ para de esa forma prevenir una postura pasiva en el proceso de toma de decisiones compartidas. ${ }^{24}$

Los varones y aquellos con capacitación en metodología obtuvieron los resultados más altos en las escalas Numérica Subjetiva y Numérica Objetiva, hallazgos identificados en otros estudios. ${ }^{25,26}$ Este resultado sugiere que la capacitación de metodología es eficaz en desarrollar y aumentar las habilidades numéricas relevantes en la práctica médica. La capacitación en metodología tuvo una respuesta dicotómica, lo cual impide la evaluación de características propias de la formación, como el tipo de entrenamiento, la duración del curso y los créditos obtenidos, lo cual es una limitación del estudio. Sin embargo, el interés de incluir esta variable fue identificar la influencia de algún tipo de capacitación en las habilidades numéricas y gráficas, pues finalmente no se podrá saber si los conceptos enseñados fueron aprendidos en capacitación al margen de sus características.

En nuestro medio, es más frecuente notar que la carrera de pregrado está mejor estructurada que las de posgrado, las cuales suelen estar basadas en la exposición del médico en entrenamiento a los pacientes de su especialidad más que en el desarrollo de competencias en metodología, investigación o comunicación. Si bien los tipos de población estudiada pueden representar un continuo en la formación médica, sexto año, séptimo año y luego la residencia, los residentes no necesariamente provienen de la misma universidad de formación en el pregrado (nuestra muestra incluye solo $10 \%$ de los residentes que cursaban el pregrado en la Universidad Peruana Cayetano Heredia), lo cual puede mostrar las diferencias en los currículos de las demás universidades respecto al entrenamiento en metodología e investigación.

Esto puede explicar la diferencia entre los resultados de los alumnos de pregrado y residentes evaluados: en la Universidad Peruana Cayetano Heredia el currículo de la carrera de medicina incluye metodología de la investigación, cursos de bioestadística y demografía (segundo año), epidemiologia y salud pública (tercer año), epidemiologia clínica (quinto año) y metodología de la investigación (quinto y sexto año). Esto finalmente redunda en que sea reconocida como una de las universidades líderes en el Perú y en Latinoamérica en investigación y una de las dos universidades con mayor producción científica en el país. ${ }^{19,27}$

Los resultados de la habilidad gráfica muestran que una elevada puntuación no solo se relacionaba con el sexo masculino sino también con la edad de los alumnos. Es posible que los alumnos más jóvenes hayan recibido capacitación mejor o diferente que les permitió desarrollar sus habilidades gráficas. Alternativamente, la diferencia entre los alumnos mayores y menores se puede deber al tiempo transcurrido entre la capacitación en metodología, la cual pudo haberse desarrollado únicamente en el pregrado y el inicio del entrenamiento como especialista. Es compatible con esta explicación que los internos de medicina tuvieron una habilidad gráfica mayor que los residentes de medicina., lo cual demuestra la heterogeneidad entre los participantes del estudio.

Este análisis exploratorio permitió establecer que los alumnos de pregrado (sexto y séptimo de la carrera de medicina) sí cuentan con conocimientos en metodología, que se traducen en mayores resultados en las escalas numéricas objetiva y subjetiva. Por ello es importante establecer que como parte de su 
formación académica, los alumnos de pregrado (sexto y séptimo) y posgrado (residentes de medicina) necesitan conocimientos en metodología y bioestadística para un mejor entendimiento y comprensión de conceptos numéricos y gráficos, esenciales para el entendimiento y comunicación en temas de salud. ${ }^{17}$ Estudios futuros deberán investigar cómo aumentar las habilidades numéricas y gráficas, especialmente en los estudiantes con problemas graves para calcular y evaluar datos relevantes.

Entre las limitaciones de nuestro estudio están que no se pudo evaluar un número mayor de alumnos de séptimo y residentes de medicina, para realizar una mayor comparación de los grupos, y que la población se restringió a una sola universidad (en los últimos años el número de facultades de medicina en Perú se ha incrementado y se requiere un estudio que abarque una muestra representativa).

En conclusión, nuestros resultados demuestran que los estudiantes de pre y posgrado de la Universidad Nacional Peruana Cayetano Heredia tuvieron habilidades numéricas y gráficas aceptables, relacionadas con factores demográficos como la edad, el sexo y con factores de formación como la capacitación en metodología.

\section{Bibliografía}

1. Rao G. Physician numeracy: essential skills for practicing evidence-based medicine. Fam Med. 2008;40(5):354-358.

2. Elwyn G, Frosch D, Thomson R, Joseph-Williams N, Lloyd A, Kinnersley $P$, et al. Shared decision making: a model for clinical practice. J Gen Intern Med. 2012;27(10):1361-1367.

3. Apter AJ, Paasche-Orlow MK, Remillard JT, Bennett IM, Ben-Joseph EP, Batista RM, et al. Numeracy and communication with patients: they are counting on us. J Gen Intern Med. 2008;23(12):2117-2124.

4. Garcia-Retamero R, Galesic M. Transparent communication of health risks: overcoming cultural differences. EE. UU.: Springer; 2013.

5. Garcia-Retamero R, Wicki B, Cokely ET, Hanson B. Factors predicting surgeons' preferred and actual roles in interactions with their patients. Health Psychol. 2014;33(8):920-928.

6. Gigerenzer G, Gaissmaier W, Kurz-Milcke E, Schwartz LM, Woloshin S. Helping doctors and patients make sense of health statistics. Psychol Sci Public Interest. 2007;8(2):53-96.
7. Wegwarth O, Schwartz LM, Woloshin S, Gaissmaier W, Gigerenzer G. Do physicians understand cancer screening statistics? A national survey of primary care physicians in the United States. Ann Intern Med. 2012;156(5):340-349.

8. Cokely ET, Galesic M, Schulz E, Ghazal S, Garcia-Retamero R. Measuring risk literacy: The Berlin Numeracy Test. Judgm Decis Mak. 2012;7(1):25-47.

9. Schapira MM, Walker CM, Cappaert KJ, Ganschow PS, Fletcher KE, McGinley $\mathrm{EL}$, et al. The numeracy understanding in medicine instrument: a measure of health numeracy developed using item response theory. Med Decis Making. 2012;32(6):851-865

10. Weller JA, Dieckmann NF, Tusler M, Mertz C, Burns WJ, Peters E. Development and testing of an abbreviated numeracy scale: a rasch analysis approach. J Behav Decis Making. 2013;26(2):198-212.

11. Garcia-Retamero R, Dhami M. Pictures speak louder than numbers: on communicating medical risks to immigrants with limited non-native language proficiency. Health Expect. 2011;14(Suppl 1):46-57.

12. Garcia-Retamero R, Cokely E. Communicating health risks with visual aids. Curr Direct Psychol Sci 2013; 22(5):392-399.

13. Lipkus I, Samsa G, Rimer B. General performance on a numeracy scale among highly educated samples. Med Decis Making. 2001;21(1):37-44.

14. Zikmund-Fisher BJ, Smith DM, Ubel PA, Fagerlin A. Validation of the subjective numeracy scale: effects of low numeracy on comprehension of risk communications and utility elicitations. Med Decis Making. 2007;27(5):663-671.

15. Fagerlin A., Zikmund-Fisher BJ, Ubel PA, Jankovic A., Derry HA, Smith DM. Measuring numeracy without a math test: development of the Subjective Numeracy Scale. Med Decis Making. 2007;27(5):672-680.

16. Galesic M, Garcia-Retamero R. Graph literacy: a cross-cultural comparison. Med Decis Making. 2011;31(3):444-457.

17. Galesic M, Garcia-Retamero R. Do low numeracy people avoid shared decision making? Health Psychol. 2011;30(3):336-341.

18. Sheridan SL, Pignone M. Numeracy and the medical student's ability to interpret data. Eff Clin Pract. 2002;5(1):35-40.

19. Consejo Nacional de Ciencia, Tecnología e Innovación Tecnológica (CONCYTEC). Produccion cientifica en Perú en base a la base de datos SCOPUS de Universidades 2015. CONCYTEC Repositorio de datos. Lima, 2016. Disponible en: http://datos.concytec.gob.pe/sites/default/files/ PRODUCCION_CIENTIFICA_SCOPUS_UNIVERSIDADES_2015.xIsx

20. McMullan M. Exploring the numeracy skills of nurses and students when performing drug calculations. Nurs Times. 2010;106(34):10-12.

21. Johnson TV, Abbasi A, Schoenberg ED, Kellum R, Speake LD, Spiker C, et al. Numeracy among trainees: are we preparing physicians for evidence-based medicine? J Surg Educ. 2014;71(2):211-215.

22. Windish DM, Huot SJ, Green ML. Medicine residents' understanding of the biostatistics and results in the medical literature. JAMA. 2007:298(9):1010-1022.

23. Garcia-Retamero R, Hoffrage, U. Visual representation of statistical information improves diagnostic inferences in doctors and their patients. Soc Sci Med. 2013;83:27-33.

24. Trevena LJ, Zikmund-Fisher BJ, Edwards A, Gaissmaier W, Galesic M, Han PK, et al. Presenting quantitative information about decision outcomes: a risk communication primer for patient decision aid developers. BMC Med Inform Decis Mak. 2013:13(Suppl 2):S7

25. Okan Y, Garcia-Retamero R, Cokely E, Maldonado A. Individual differences in graph literacy: overcoming denominator neglect in risk comprehension. J Behav Decis Mak. 2012;25(4):390-401.

26. Schwartz LM, Woloshin S, Black WC, Welch HG. The role of numeracy in understanding the benefit of screening mammography. Ann Intern Med. 1997;127(11):966-972.

27. Huamani $C$, Mayta-Tristán $P$. Producción científica peruana en medicina y redes de colaboración, análisis del Science Citation Index 2000-2009. Rev Peru Med Exp Salud Publica. 2010;27(3):315-325. 